\title{
Particle Swarm Optimisation Aided MIMO Multiuser Transmission Designs
}

\author{
W. Yao, S. Chen*, and L. Hanzo \\ School of Electronics and Computer Science, University of Southampton, Southampton S017 1BJ, UK
}

\begin{abstract}
Bio-inspired computational methods have found wide-ranging applications in signal processing and other walks of engineering. The main attraction of adopting bio-inspired computational intelligence algorithms is that they may facilitate global or near global optimal designs with affordable computational costs. In this contribution, particle swarm optimisation (PSO) is invoked for designing optimal multiuser transmission (MUT) schemes for multiple-input multiple-output communication. Specifically, we consider the minimum bit-error-rate (MBER) linear MUT using PSO and we design a PSO aided MBER generalised vector precoding for nonlinear MUT. These PSO aided MUT techniques compare favourably with the state-of-the-art conventional schemes, in terms of performance and complexity.
\end{abstract}

Keywords: Bio-Inspired Computation, Evolutionary Computation, Particle Swarm Optimisation, Multiple-Input Multiple-Output Communication, Multiuser Transmission, Precoding, Vector Precoding.

\section{INTRODUCTION}

Bio-inspired computational intelligence methods, such as the generic algorithm (GA), ant colony optimisation (ACO) and particle swarm optimisation (PSO), have found ever-increasing applications in all walks of engineering, especially communication signal processing, where attaining global or near global optimal solutions at affordable computational costs are critical. The Communication Research Group at the University of Southampton has a long and successful record in applying the GA and ACO in multiuser detection (MUD) applications. ${ }^{1-9}$ This contribution focuses on the optimal multiuser transmission (MUT) designs for multiple-input multiple-output (MIMO) communication systems with the aid of PSO. PSO constitutes a population based stochastic optimisation technique, inspired by the social behaviour of bird flocks or fish schools. ${ }^{10,11}$ The algorithm commences with random initialisation of a swarm of individuals, referred to as particles, within the problem's search space. Each particle then gradually adjusts its trajectory with the aid of cognitive information (its own best location) and social information (the best position of the entire swarm) at each optimisation iteration. PSO is simple to implement, has ability to rapidly converge and is capable of steering clear of local minima. Recently, PSO has become popular and has been

*Author to whom correspondence should be addressed. applied to a variety of engineering applications. ${ }^{11-26}$ In particular, many results have been reported on the application of PSO techniques to MUD. ${ }^{27-31}$ By contrast, few work has used the PSO to assist the design of MUT. ${ }^{32}$

The family of well-studied MUD is designed for mitigating the multiuser interference at the base station (BS), which is imposed by the uplink transmissions of the mobile stations (MSs) to the BS. In the downlink of the MIMO system, the BS, equipped with multiple transmitting antennas, communicates with single-receive-antenna mobile stations (MSs). Simple low-complexity MSs are incapable of performing sophisticated cooperative MUD to mitigating the multiuser interference. In order to facilitate the employment of a low-complexity, high-powerefficiency single-user receiver, the transmitted multiuser downlink signals may be pre-processed at the BS, leading to the appealing concept of MUT. ${ }^{33,34}$ More explicitly, the MUT requires the knowledge of each user's unique channel impulse response (CIR) for differentiating the different users' transmissions. For time division duplex systems, the uplink CIRs measured at the BS may be exploited for subsequent downlink pre-processing owing to the channel's reciprocity. However, the CIR has to be explicitly fed back from the MS's receivers to the BS transmitter in frequency division duplex systems, where the uplink and downlink CIRs are different. MUT schemes can be divided into the two groups, namely, linear MUT schemes and nonlinear MUT schemes. 
A well-known linear MUT design is based on the minimum mean square error (MMSE) criterion, ${ }^{35}$ which has appealing simplicity but is limited by its achievable bit error rate (BER) performance. The optimal linear MUT design has been developed based on the minimum BER (MBER) criterion. ${ }^{36-40}$ The linear MBER-MUT design invokes a constrained nonlinear optimisation and the sequential quadratic programming (SQP) algorithm ${ }^{41}$ is typically used to obtain the precoder's coefficients. However, the computational complexity of the SQP based MBER-MUT solution can be excessive for high-rate systems. In this contribution, we invoke the PSO to solve the constrained nonlinear optimisation problem for the MBER-MUT, and we show that the PSO aided MBERMUT scheme provides improved performance in comparison to the conventional MMSE-MUT scheme, while imposing a significantly reduced complexity compared to the state-of-the-art SQP based MBER-MUT design.

Nonlinear MUT techniques are capable of approaching the rate region of dirty paper coding. ${ }^{42}$ Specifically, the vector precoding (VP) algorithm ${ }^{43-47}$ is capable of outperforming any linear pre-processing technique, in terms of the system's achievable BER, at the expense of an increased complexity. The performance gain of a VP based MUT over a linear MUT scheme is particularly significant in the rank-deficient senario where the number of the BS transmitter antennas is smaller than the number of MSs supported. In the VP precoder, the data vector is perturbed by a perturbation vector, which is then multiplied by the precoding matrix to generate the effective symbol vector to be transmitted. The design is then to determine the precoding matrix and the perturbation vector separately. The existing powerful VP design is the nonlinear MMSE VP scheme. ${ }^{45}$ We propose to generate the effective symbol vector directly by minimising the BER criterion. This generalised MBER VP design is a challenging non-convex optimisation, and we adopt the efficient PSO algorithm to solve this design. The proposed PSO aided generalised MBER VP is shown to dramatically outperform the existing powerful nonlinear MMSE VP benchmark, ${ }^{45}$ at a cost of slightly increased computational complexity.

The following notational conventions are adopted in this contribution. Boldface capitals and lower-case letters stand for matrices and vectors, respectively. Furthermore, ()$^{T}$ represents the transpose operator, while \|\|$^{2}$ and || denote the norm and the magnitude operators, respectively. $E$ [] denotes the expectation operator, while $\mathfrak{R}[]$ and $\Im[]$ represent the real and imaginary parts, respectively. Finally, $j=\sqrt{-1}$.

\section{PARTICLE SWARM OPTIMISATION}

Consider the generic optimisation task defined as follows

$$
\begin{gathered}
\mathbf{U}_{\text {opt }}=\arg \min _{\mathbf{U}} F(\mathbf{U}) \\
\text { s.t. } \mathbf{U} \in \mathbf{U}^{N \times M}
\end{gathered}
$$

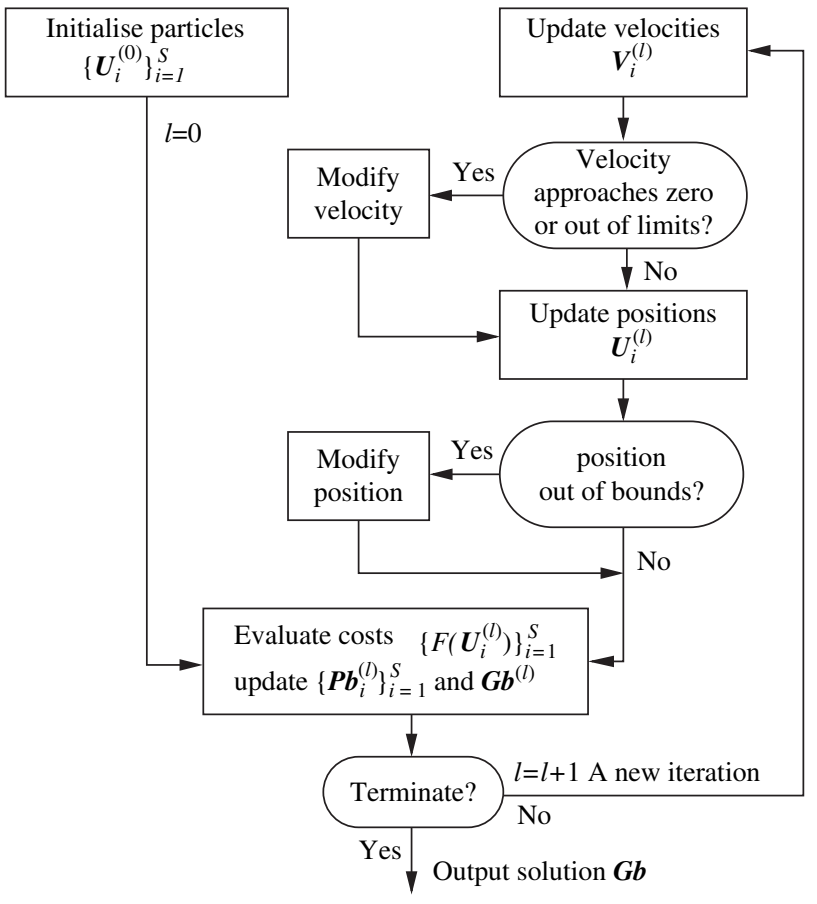

Fig. 1. Flowchart of the PSO algorithm.

where $F()$ is the cost function, $\mathbf{U}$ is a $N \times M$ complexvalued parameter matrix to be optimised, and

$$
\mathrm{U}=\left[-U_{\max }, U_{\max }\right]+j\left[-U_{\max }, U_{\max }\right]
$$

defines the search range for each element of $\mathbf{U}$. The flowchart of the PSO algorithm is given in Figure 1. A swarm of particles, $\left\{\mathbf{U}_{i}^{(l)}\right\}_{i=1}^{S}$, that represent potential solutions are evolved in the search space $\mathrm{U}^{N \times M}$, where $S$ is the swarm size and index $l$ denotes the iteration step. A PSO algorithm typically consists of the four blocks, namely, the swarm initialisation, the swarm evaluation, the swarm updating and the termination test, as can be seen in Figure 1. The algorithm is now summarised.

\subsection{PSO Algorithm}

(a) Initialisation. Set $l=0$ and randomly generate the initial particles, $\left\{\mathbf{U}_{i}^{(l)}\right\}_{i=1}^{S}$, in the search space $\mathbf{U}^{N \times M}$.

(b) Evaluation. Each particle $\mathbf{U}_{i}^{(l)}$ has an associated cost $F\left(\mathbf{U}_{i}^{(l)}\right)$, and it remembers its best position visited so far, denoted as $\mathbf{P} \mathbf{b}_{i}^{(l)}$, which provides the cognitive information. Every particle also knows the best position visited so far among the entire swarm, denoted as $\mathbf{G} \mathbf{b}^{(l)}$, which provides the social information. The cognitive information $\left\{\mathbf{P} \mathbf{b}_{i}^{(l)}\right\}_{i=1}^{S}$ and the social information $\mathbf{G b}^{(l)}$ are updated at each iteration given the new cost information $\left\{F\left(\mathbf{U}_{i}^{(l)}\right)\right\}_{i=1}^{S}$. (c) Update. Each particle $\mathbf{U}_{i}^{(l)}$ has a velocity, denoted as $\mathbf{V}_{i}^{(l)}$, to direct its "flying" or search. The velocity and position of the $i$ th particle are updated in each iteration according to:

$$
\mathbf{V}_{i}^{(l+1)}=\xi * \mathbf{V}_{i}^{(l)}+c_{1} * \varphi_{1} *\left(\mathbf{P} \mathbf{b}_{i}^{(l)}-\mathbf{U}_{i}^{(l)}\right)
$$

J. Comput. Theor. Nanosci. 9, 1-10, 2012 


$$
\begin{aligned}
& +c_{2} * \varphi_{2} *\left(\mathbf{G} \mathbf{b}^{(l)}-\mathbf{U}_{i}^{(l)}\right) \\
\mathbf{U}_{i}^{(l+1)}= & \mathbf{U}_{i}^{(l)}+\mathbf{V}_{i}^{(l+1)}
\end{aligned}
$$

where $\xi$ is the inertia weight, $c_{1}$ and $c_{2}$ are the two acceleration coefficients, while $\varphi_{1}=\operatorname{rand}()$ and $\varphi_{2}=\operatorname{rand}()$ denotes the two random variables uniformly distributed in $(0,1)$.

In order to avoid excessive roaming of particles beyond the search space, a velocity space $\mathrm{V}^{N \times M}$ with

$$
\mathrm{V}=\left[-V_{\max }, V_{\max }\right]+j\left[-V_{\max }, V_{\max }\right]
$$

is imposed so that each element of $\mathbf{V}_{i}^{(l+1)}$ is within the search range $\mathrm{V}$ defined in (6). Furthermore, if an element of $\mathbf{V}_{i}^{(l+1)}$ approaches zero, it may be randomly reinitialised within the velocity range $\mathrm{V}$. Similarly, if a particle $\mathbf{U}_{i}^{(l+1)}$ moves to outside the search space, it is moved back inside $\mathrm{U}^{N \times M}$ to a random position.

(d) Termination. If the maximum number of iterations, $I_{\max }$, is reached, terminate with the solution $\mathbf{U}_{\mathrm{opt}}=\mathbf{G b}^{\left(I_{\max }\right)}$; otherwise, set $l=l+1$ and go to Step $(b)$.

\subsection{PSO Algorithmic Parameters}

The search limit $U_{\max }$ is specified by the problem considered, while the velocity limit $V_{\max }$ is typically related to $U_{\max }$. The value of $V_{\max }$ may also be determined empirically. Three common choices of the inertia weight are $\xi=$ 0 , setting $\xi$ to a small positive constant, and $\xi=\operatorname{rand}()$. The time varying acceleration coefficients (TVAC), ${ }^{12}$ in which $c_{1}$ is reduced from 2.5 to 0.5 and $c_{2}$ varies from 0.5 to 2.5 during the iterative procedure according to

$$
\begin{aligned}
& c_{1}=(0.5-2.5) * l / I_{\max }+2.5 \\
& c_{2}=(2.5-0.5) * l / I_{\max }+0.5
\end{aligned}
$$

usually works well. The reason for good performance of this TVAC mechanism can be explained as follows. At the initial stages, a large cognitive component and a small social component help particles to wander around or better exploit the search space, avoiding local minima. In the later stages, a small cognitive component and a large social component help particles to converge quickly to a global minimum.

Let the complexity of one cost function evaluation be $C_{\text {single }}$. With the swarm size $S$ and assuming that the algorithm converges in $I_{\max }$ iterations, the number of cost function evaluations is $N_{\text {total }}=S \times I_{\max }$, and the complexity of the algorithm is given by

$$
C_{\mathrm{PSO}}=N_{\text {total }} \times C_{\text {single }}=S \times I_{\text {max }} \times C_{\text {single }}
$$

Appropriate values for $S$ and $I_{\max }$ can be chosen to ensure that the algorithm converges to the optimal solution with a minimum computational complexity. In our applications, $S$ is found in the range of 20 to 40 , while the corresponding $I_{\max }$ in the range of 25 to 40 . Therefore, an attraction of PSO is that the algorithm can be easily tuned by choosing suitable $S$ and $I_{\max }$ to attain an optimal solution with a small $N_{\text {total }}$.

\section{LINEAR MBER MUT DESIGN}

Our first application involves the PSO aided linear MBER MUT design for MIMO communication.

\subsection{Linear MUT System Model}

The linear MUT-aided MIMO system is depicted in Figure 2, where the BS equipped with $N$ transmitting antennas communicates with $K$ MSs, each employing a single-receiver antenna. In this downlink communication, simple MSs are unable to perform sophisticated MUD to combat multiuser interference. A solution is to let the BS do MUT. The MUT scheme shown in Figure 2 is linear as the BS uses linear precoding to preprocess the transmitted multiuser downlink signals. The model of this linear MUT assisted MIMO system is now explained.

The information symbol vector transmitted is given by $\mathbf{x}=\left[\begin{array}{llll}x_{1} & x_{2} \cdots x_{K}\end{array}\right]^{T}$, where $x_{k}$ denotes the transmitted symbol to the $k$ th MS, and we further assume that $x_{k}$ takes the value from the 4-QAM symbol set

$$
\mathscr{S}=\left\{ \pm \frac{1}{2} \pm j \frac{1}{2}\right\}
$$

Extension to higher-order QAM schemes can be achieved by following the approach for MUD as shown in. ${ }^{48}$ The MUT's $N \times K$ precoder matrix $\mathbf{P}$ is defined by

$$
\mathbf{P}=\left[\begin{array}{lll}
\mathbf{p}_{1} & \mathbf{p}_{2} \cdots \mathbf{p}_{K}
\end{array}\right]
$$

where $\mathbf{p}_{k}, 1 \leq k \leq K$, is the precoder's coefficient vector for preprocessing the $k$ th user's data stream. Given a fixed total transmit power $\mathrm{E}_{\mathrm{T}}$ at the $\mathrm{BS}$, an appropriate scaling factor should be used to fullfill this transmit power constraint, which is defined as $\alpha=\sqrt{\mathrm{E}_{\mathrm{T}} /\|\mathbf{P x}\|^{2}}$.

At the receiver, the reciprocal of $\alpha$ is used to scale the received signal to ensure unity-gain transmission. The MIMO channel matrix $\mathbf{H}$ is given by

$$
\mathbf{H}=\left[\mathbf{h}_{1} \mathbf{h}_{2} \cdots \mathbf{h}_{K}\right]
$$

where $\mathbf{h}_{k}=\left[h_{1, k} h_{2, k} \cdots h_{N, k}\right]^{T}, 1 \leq k \leq K$, is the $k$ th user's spatial signature. The channel taps $h_{i, k}$ for $1 \leq k \leq K$ and $1 \leq i \leq N$ are independent of each other and obey the complex-valued Gaussian distribution with $E\left[\left|h_{i, k}\right|^{2}\right]=1$. The additive white Gaussian noise vector $\mathbf{n}$ is defined by $\mathbf{n}=\left[\begin{array}{llll}n_{1} & n_{2} \cdots n_{K}\end{array}\right]^{T}$, where $n_{k}, 1 \leq k \leq K$, is a complexvalued Gaussian white noise with $E\left[\left|n_{k}\right|^{2}\right]=2 \sigma_{n}^{2}=\mathrm{N}_{\mathrm{o}}$. The signal-to-noise ratio (SNR) of the system is defined as $\mathrm{SNR}=\mathrm{E}_{\mathrm{b}} / \mathrm{N}_{\mathrm{o}}$, where $\mathrm{E}_{\mathrm{b}}=\mathrm{E}_{\mathrm{T}} /\left(N \log _{2} M\right)$ is the energy 


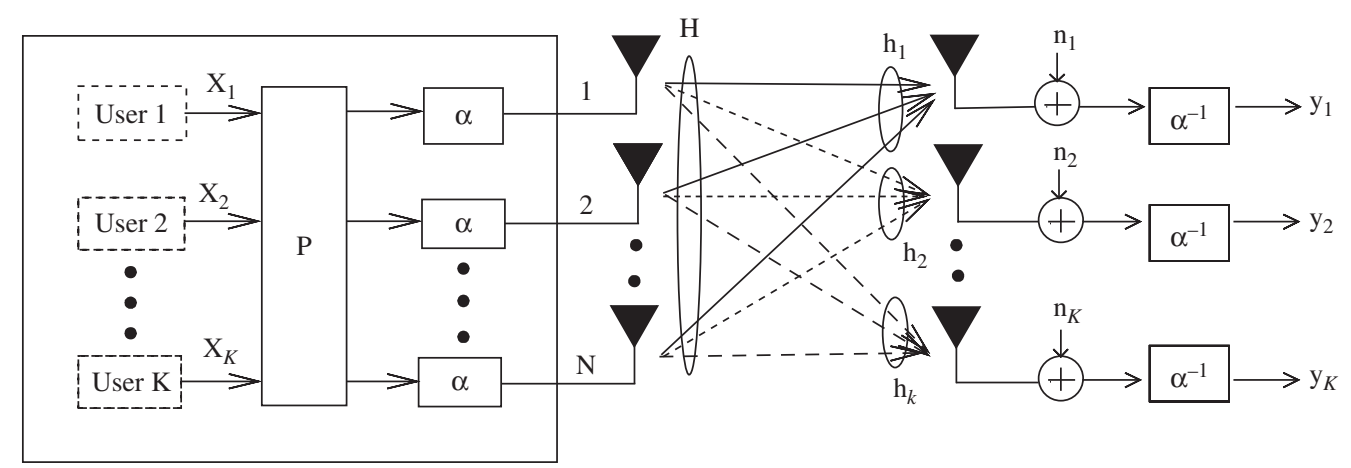

Fig. 2. MUT-aided MIMO system with linear precoding, where the BS employs $N$ transmitter antennas to communicate with $K$ single-receiver-antenna mobile devices.

per bit per antenna for $M$-ary modulation. For the 4-QAM case $M=4$. The system model is given by

$$
\mathbf{y}=\mathbf{H}^{T} \mathbf{P} \mathbf{x}+\alpha^{-1} \mathbf{n}
$$

where $\mathbf{y}=\left[\begin{array}{ll}y_{1} & y_{2} \cdots y_{K}\end{array}\right]^{T}$ denotes the received signal vector.

The task of the generic linear MUT design can now be stated. Given the MIMO channel matrix $\mathbf{H}$, the information symbol vector to be transmitted $\mathbf{x}$ and the statistics of the noise vector $\mathbf{n}$, design the precoding matrix $\mathbf{P}$ so that $y_{k}$, $1 \leq k \leq K$, constitutes sufficient statistics for the $k$ th MS to detect the transmitted data symbol $x_{k}$. This ensures that the $k$ th MS equipped with a conventional matched filter can simply estimate $x_{k}$ by quantising $y_{k}$.

The best-known linear MUT design is the MMSE solution, ${ }^{34,35}$ which is computationally very simple but is non-optimal in terms of its BER performance. Previous works $^{36-40}$ have developed the optimal MBER linear MUT solution, which is now summarised.

\subsection{Linear MBER MUT Design}

Given the 4-QAM symbol vector $\mathbf{x}$, the average BER of the in-phase component of $\mathbf{y}$ at the receivers is ${ }^{38}$

$$
P_{e_{I}, \mathbf{x}}=\frac{1}{K} \sum_{k=1}^{K} Q\left(\frac{\operatorname{sgn}\left(\Re\left[x_{k}\right]\right) \Re\left[\mathbf{h}_{k}^{T} \mathbf{P x}\right]}{\sigma_{n}}\right)
$$

where $Q(\bullet)$ is the standard Gaussian error function. Similarly, given the symbol vector $\mathbf{x}$, the average BER of the quadrature-phase component of $\mathbf{y}$ is

$$
P_{e_{Q}, \mathbf{x}}=\frac{1}{K} \sum_{k=1}^{K} Q\left(\frac{\operatorname{sgn}\left(\Im\left[x_{k}\right]\right) \Im\left[\mathbf{h}_{k}^{T} \mathbf{P x}\right]}{\sigma_{n}}\right)
$$

Thus, the resultant BER for the specific 4-QAM symbol $\mathbf{x}$ is

$$
P_{e, \mathbf{x}}(\mathbf{P})=\left(P_{e_{I}, \mathbf{x}}(\mathbf{P})+P_{e_{Q}, \mathbf{x}}(\mathbf{P})\right) / 2
$$

Therefore, the MBER-MUT design is defined as the solution of the following constrained optimisation

$$
\begin{gathered}
\mathbf{P}_{\mathrm{MBER}, \mathbf{x}}=\arg \min _{\mathbf{P}} P_{e, \mathbf{x}}(\mathbf{P}) \\
\text { s.t. }\|\mathbf{P x}\|^{2}=\mathrm{E}_{\mathrm{T}}
\end{gathered}
$$

This constrained nonlinear optimisation is typically solved by an iterative gradient based algorithm known as the SQP. ${ }^{37-40}$ The computational complexity of this SQPbased linear MBER MUT design can be found in. ${ }^{32,40}$ The SQP based design has a high computational complexity. Therefore, for practical high rate systems, it may be difficult to implement this SQP based MBER linear MUT solution.

Our objective is to use the PSO algorithm as an alternative to the SQP algorithm in order to achieve a lowcomplexity linear MBER MUT design. Following the approach of, ${ }^{32}$ a penalty function approach is adopted to convert the constrained optimisation (16) into an unconstrained one which automatically meets the transmit power constraint. Define the cost function as

$$
F(\mathbf{P})=P_{e, \mathbf{x}}(\mathbf{P})+G_{\mathbf{x}}(\mathbf{P})
$$

with the penalty function given by

$$
G_{\mathbf{x}}(\mathbf{P})= \begin{cases}0, & \|\mathbf{P x}\|^{2}-\mathrm{E}_{\mathrm{T}} \leq 0 \\ \lambda\left(\|\mathbf{P x}\|^{2}-\mathrm{E}_{\mathrm{T}}\right), & \|\mathbf{P x}\|^{2}-\mathrm{E}_{\mathrm{T}}>0\end{cases}
$$

where the penalty factor $\lambda>0$ should be chosen appropriately so that the MBER-MUT design (16) becomes the following unconstrained optimisation

$$
\mathbf{P}_{\mathrm{MBER}, \mathbf{x}}=\arg \min _{\mathbf{P}} F(\mathbf{P})
$$

where the precoding matrix $\mathbf{P} \in \mathrm{U}^{N \times K}$. A suitable $\lambda$ value is not difficult to choose at all, since the BS transmitter has the knowledge of $\mathbf{H}, \mathbf{x}$ and the covariance matrix of $\mathbf{n}$. The PSO algorithm described in Section 2 can readily be adopted to solve this optimisation problem.

For the system introduced in Subsection 3.1, our empirical results suggest that the search limit can be set to $U_{\max }=1$ while the velocity limit can be set to $V_{\max }=1$. We also remove the influence of the previous velocity by setting $\xi=0$, which works well for this application. In Step (a), one of the initial particles is set to the MMSEMUT solution. ${ }^{35}$ The two PSO algorithmic parameters that determine performance and complexity are then the swarm 
size $S$ and the maximum iteration number $I_{\max }$. The analysis and empirical results given in ${ }^{32}$ show that, by choosing appropriate values for $S$ and $I_{\max }$, the PSO aided design achieves the same optimal MBER MUT solution as the SQP based one does, but at a significantly reduced computational complexity.

\subsection{Simulation Results}

The MIMO system considered employed $N=4$ transmitting antennas at the BS to communicate with $K=4 \mathrm{MSs}$. All the simulation results were obtained by averaging over 100 channel realisations. An appropriate swarm size was found to be $S=20$ empirically. The maximum number of iterations, $I_{\max }$, was so chosen such that the PSO-based linear MBER-MUT algorithm with the chosen $I_{\max }$ and $S=$ 20 arrived at the same MBER performance also achieved by the SQP-based MBER-MUT design. The value of $I_{\max }$ was in the range of 20 to 30 , depending on the value of the channel SNR. Figure 3 compares the BER performance of the linear MMSE-MUT scheme with those of the PSOaided linear MBER-MUT design, where it can be seen that the PSO-aided MBER-MUT design achieved an SNR gain of $4.5 \mathrm{~dB}$ over the MMSE-MUT benchmark at the target BER of $10^{-4}$.

We now explain why the swarm size was chosen to be $S=20$ in this application. Given $\mathrm{SNR}=15 \mathrm{~dB}$, convergence performance of the PSO-aided linear MBER-MUT scheme with different swarm sizes are plotted in Figure 4. It is clear from Figure 4 that $S=10$ was insufficient for the PSO to attain the optimal solution, while the PSO algorithm with $S=20,30$ and 40 all converged to the optimal solution after $I_{\max }=30,25$ and 20, respectively. The complexity $C_{\mathrm{PSO}}$ of the PSO-aided linear MBER-MUT scheme for these three values of $S$ are listed in Table I. We can

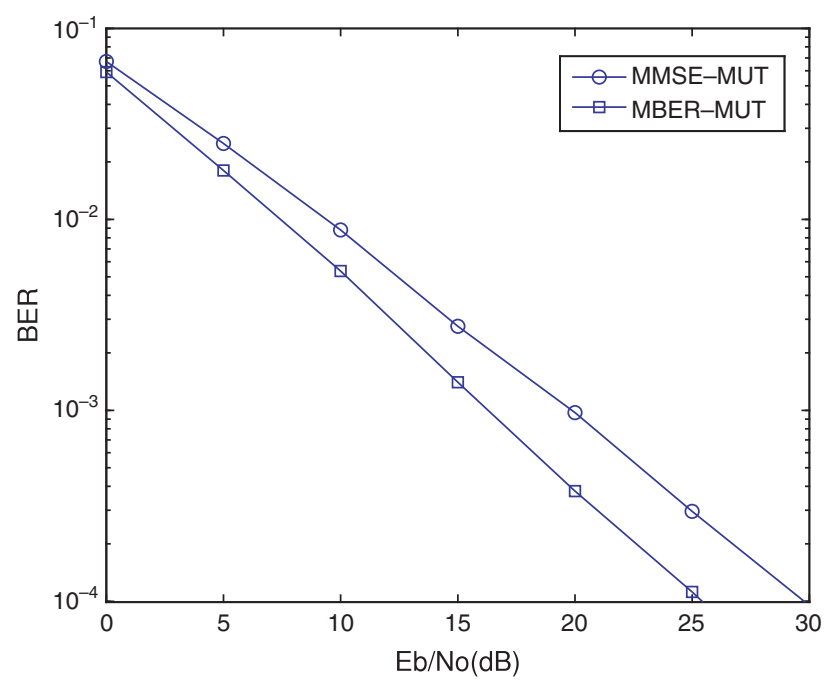

Fig. 3. BER performance of the PSO-aided linear MBER-MUT design for the $4 \times 4$ MIMO system, in comparison with the benchmark MMSEMUT.

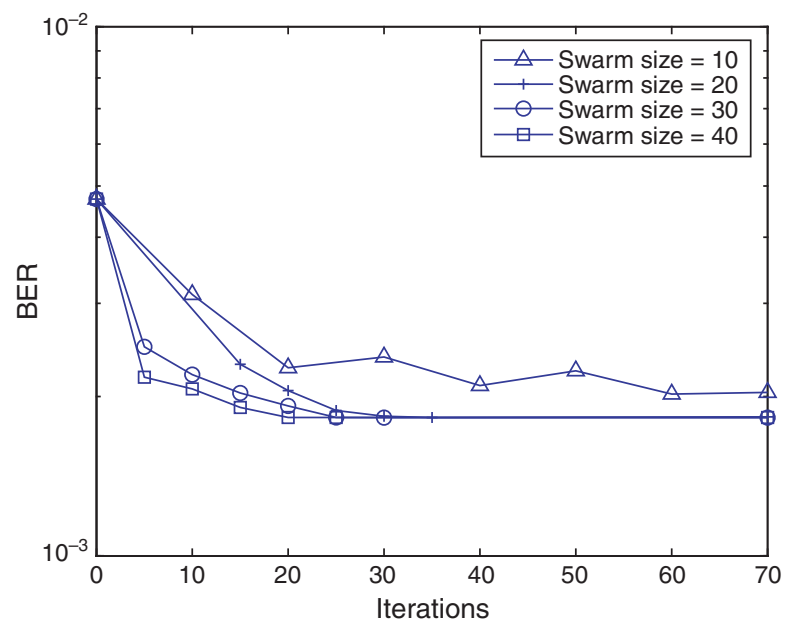

Fig. 4. Convergence of the PSO MBER-MUT scheme with different swarm sizes for the $4 \times 4$ MIMO system given $\mathrm{SNR}=15 \mathrm{~dB}$.

Table I. Complexity (Flops) of the PSO aided linear MBER-MUT design with different swarm sizes for the $4 \times 4$ MIMO system given $\mathrm{SNR}=15 \mathrm{~dB}$.

\begin{tabular}{lrrr}
\hline Swarm size $S$ & 20 & 30 & 40 \\
Iterations $I_{\max }$ & 30 & 25 & 20 \\
$C_{\text {PSO }}$ (Flops) & 402,840 & 503,450 & 536,960 \\
\hline
\end{tabular}

see that the choice of $S=20$ was optimal for this case, in terms of computational complexity.

As stated previously, both the PSO-aided and SQPbased schemes achieved the same optimal linear MBERMUT performance. Computational complexity of the both schemes were next compared. Figure 5 compares the convergence performance of the SQP and PSO based schemes, operating at the SNR values of $10 \mathrm{~dB}$ and $15 \mathrm{~dB}$, respectively. It can be seen from Figure 5 that in the case of $\mathrm{SNR}=10 \mathrm{~dB}$, the SQP and PSO algorithms converged to the optimal solution after 70 and 20 iterations, respectively,

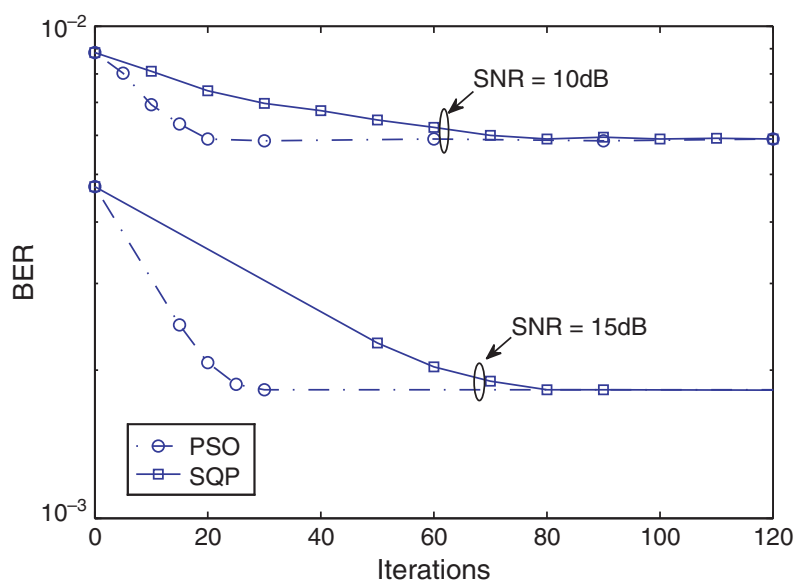

Fig. 5. Convergence performance comparison of the PSO-aided and SQP-based MBER-MUT schemes for the $4 \times 4$ MIMO system given two SNR values. 
Table II. Complexity (Flops) and recorded run time (s) comparison of the PSO and SQP aided linear MBER-MUT designs for the $4 \times 4$ MIMO system given two SNR values.

\begin{tabular}{lll}
\hline (SNR $=10 \mathrm{~dB})$ & $\mathrm{SQP}$ & $\mathrm{PSO}$ \\
\hline Iterations & 70 & 20 \\
Complexity (Flops) & $3,180,170$ & 268,560 \\
Run time (s) & 7412.1 & 664.9 \\
(SNR=15 dB) & $\mathrm{SQP}$ & $\mathrm{PSO}$ \\
Iterations & 80 & 30 \\
Complexity (Flops) & $3,634,480$ & 402,840 \\
Run time (s) & 8457.3 & 957.4 \\
\hline
\end{tabular}

while at $\mathrm{SNR}=15 \mathrm{~dB}$, the $\mathrm{SQP}$ and $\mathrm{PSO}$ algorithms arrived at the optimal solution after 80 and 30 iterations, respectively. The computational complexity and recorded run times for the two designs are listed in Table II. It can be seen from Table II that the PSO-aided linear MBER-MUT design imposed an approximately twelve times lower complexity than the SQP counterpart at SNR= $10 \mathrm{~dB}$, while it imposed an approximately nine times lower complexity than the SQP counterpart for the SNR value of $15 \mathrm{~dB}$.

\section{NONLINEAR MBER MUT DESIGN}

Our second application considers the PSO aided nonlinear MBER generalised VP design for MIMO communication.

\subsection{Generic VP System Model}

The nonlinear MUT-aided MIMO system is depicted in Figure 6, where the BS employs $N$ transmitter antennas to communicate with $K$ single-antenna MS receivers. Again this is the downlink scenario, where the BS employs MUT to combat multiuser interference. The MUT scheme shown in Figure 6, however, is nonlinear as the BS uses nonlinear VP to preprocess the transmitted multiuser downlink signals and each MS receiver employs a modulo device. This nonlinear MUT scheme is capable of outperforming any linear MUT scheme, particularly for the rank-deficient systems where the number of the BS transmitter antennas is smaller than the number of the MS receivers supported, i.e., $N<K$. This significant enhanced performance is of course achieved at the cost of higher computational complexity. The model of this nonlinear MUT assisted MIMO system is now explained.

The channel matrix $\mathbf{H}$, the information symbol vector $\mathbf{x}$, and the noise vector $\mathbf{n}$ are as defined in Subsection 3.1. Given $\mathbf{x}, \mathbf{H}$ and the statistics of $\mathbf{n}$, the generic VP generates the continuous-valued effective symbol vector $\mathbf{d}=$ $\left[\begin{array}{ll}d_{1} & d_{2} \\ \cdots & d_{N}\end{array}\right]^{T}$, in order to mitigate multiuser interference. In a conventional VP design, $\mathbf{d}$ is expressed as

$$
\mathbf{d}=\mathbf{P}(\mathbf{x}+\boldsymbol{\omega})
$$

where $\mathbf{P}$ is the $N \times K$ precoding matrix and $\boldsymbol{\omega}$ the $K$-element discrete-valued perturbation vector. Various VP schemes determine $\mathbf{P}$ and $\boldsymbol{\omega}$ separately based on different criteria. In particular, the powerful MMSE VP scheme $^{45}$ determines the MMSE solution for $\mathbf{P}$ and seeks $\boldsymbol{\omega}$ based on the MMSE criterion. This MMSE VP design represents a state-of-the-art nonlinear MUT scheme. Again, given a fixed total transmit power $\mathrm{E}_{\mathrm{T}}$ at the BS, an appropriate scaling factor is used to fullfill this transmit power constraint, which is defined as $\alpha=\sqrt{\mathrm{E}_{\mathrm{T}} /\|\mathbf{d}\|^{2}}$.

At each MS receiver, the reciprocal of the scaling factor, namely $\alpha^{-1}$, is used to scale the received signal in order to maintain a unity-gain transmission. The received signal vector $\hat{\mathbf{y}}=\left[\begin{array}{lll}\hat{y}_{1} & \hat{y}_{2} \cdots \hat{y}_{K}\end{array}\right]^{T}$ before the modulo operation is given by

$$
\hat{\mathbf{y}}=\mathbf{H}^{T} \mathbf{d}+\alpha^{-1} \mathbf{n}
$$

The modulo operation invoked for $\hat{y}_{k}$ is described by

$$
\bmod _{\tau}\left(\hat{y}_{k}\right)=\hat{y}_{k}-\left\lfloor\frac{\Re\left[\hat{y}_{k}\right]+\tau / 2}{\tau}\right\rfloor \tau-j\left\lfloor\frac{\Im\left[\hat{y}_{k}\right]+\tau / 2}{\tau}\right\rfloor \tau
$$

where $1 \leq k \leq K,\lfloor\bullet\rfloor$ denotes the integer floor operator, and $\tau$ is a positive number determined by the modulation constellation employed. The received signal

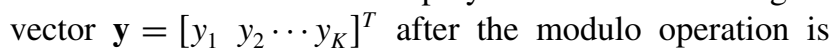
given by

$$
\mathbf{y}=\bmod _{\tau}(\hat{\mathbf{y}})
$$

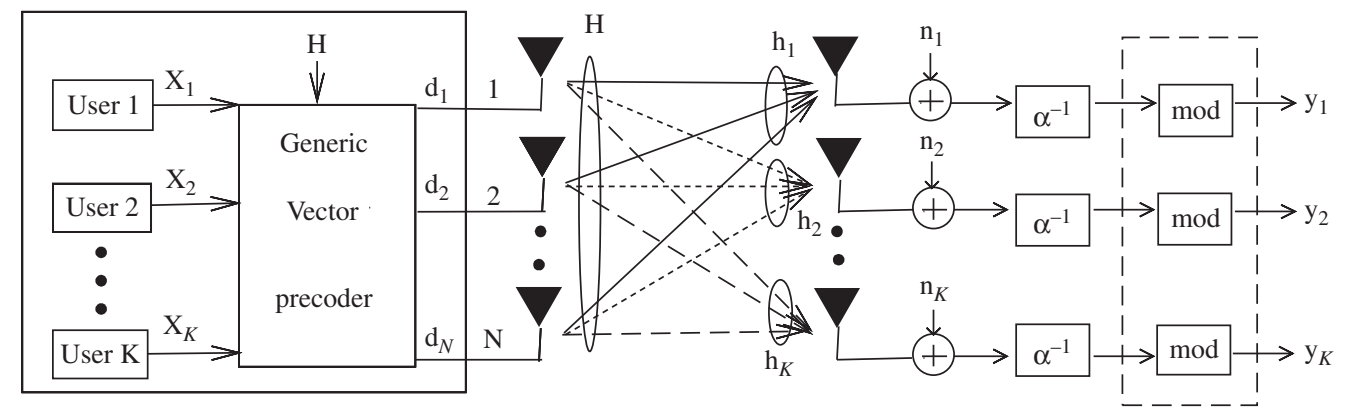

Fig. 6. MUT-aided MIMO system with nonlinear VP, where the BS employs $N$ transmit antennas to communicate with $K$ MSs each equipped with a modulo device. 
and $y_{k}, 1 \leq k \leq K$, constitutes sufficient statistics for the $k$ th MS to detect the transmitted information data symbol $x_{k}$. The authors of ${ }^{44}$ suggested to choose $\tau$ according to

$$
\tau=2\left(|c|_{\max }+\Delta / 2\right)
$$

where $|c|_{\max }$ is the largest distance of the modulated symbols to the real or imaginary axis, and $\Delta$ is the spacing between the constellation points. For the 4-QAM constellation (9), $|c|_{\max }=\frac{1}{2}$ and $\Delta=1$, which leads to $\tau=2$ according to (24). The modulo operator (22) maps the received signal, $\Re\left[\hat{y}_{k}\right]$ and $\Im\left[\hat{y}_{k}\right]$, into the interval $[-\tau / 2, \tau / 2)$.

\subsection{MBER Generalised VP Design}

Our novel VP scheme, however, does not determine $\mathbf{P}$ and $\boldsymbol{\omega}$ separately, as a conventional VP design does. Rather it directly determines $\mathbf{d}$. Therefore, we refer to this novel VP design as the generalised VP scheme. Furthermore, our design obtains $\mathbf{d}$ by minimising the MBER criterion. For notational simplicity, we again restrict to the 4-QAM constellation (9). Extension to a higher-order QAM constellation can be achieved by considering the minimum symbol error rate criterion, as in the MUD case. ${ }^{48}$

The BER encountered at the output of the receiver after the modulo operation for the in-phase component of user $k$ can be expressed as ${ }^{49}$

$$
\begin{aligned}
P_{e_{I}, k}(\mathbf{d}) \approx & Q\left(\frac{c_{R}^{(k)}+3 \tau}{\alpha^{-1} \sigma_{n}}\right)+Q\left(\frac{-\frac{5 \tau}{2}-c_{R}^{(k)}}{\alpha^{-1} \sigma_{n}}\right) \\
& -Q\left(\frac{-2 \tau-c_{R}^{(k)}}{\alpha^{-1} \sigma_{n}}\right)+Q\left(\frac{-\frac{3 \tau}{2}-c_{R}^{(k)}}{\alpha^{-1} \sigma_{n}}\right) \\
& -Q\left(\frac{-\tau-c_{R}^{(k)}}{\alpha^{-1} \sigma_{n}}\right)+Q\left(\frac{-\frac{\tau}{2}-c_{R}^{(k)}}{\alpha^{-1} \sigma_{n}}\right) \\
& -Q\left(\frac{-c_{R}^{(k)}}{\alpha^{-1} \sigma_{n}}\right)+Q\left(\frac{\frac{\tau}{2}-c_{R}^{(k)}}{\alpha^{-1} \sigma_{n}}\right) \\
& -Q\left(\frac{\tau-c_{R}^{(k)}}{\alpha^{-1} \sigma_{n}}\right)+Q\left(\frac{\frac{3 \tau}{2}-c_{R}^{(k)}}{\alpha^{-1} \sigma_{n}}\right) \\
& -Q\left(\frac{2 \tau-c_{R}^{(k)}}{\alpha^{-1} \sigma_{n}}\right)+Q\left(\frac{\frac{5 \tau}{2}-c_{R}^{(k)}}{\alpha^{-1} \sigma_{n}}\right) \\
& -Q\left(\frac{3 \tau-c_{R}^{(k)}}{\alpha^{-1} \sigma_{n}}\right)
\end{aligned}
$$

where $c_{R}^{(k)}=\operatorname{sgn}\left(\Re\left[x_{k}\right]\right) \Re\left[\mathbf{h}_{k}^{T} \mathbf{d}\right]$. Hence, the average BER of the in-phase component of $\mathbf{y}$ is given by

$$
P_{e_{I}, \mathbf{x}}(\mathbf{d})=\frac{1}{K} \sum_{k=1}^{K} P_{e_{I}, k}(\mathbf{d})
$$

Similarly, let $c_{I}^{(k)}=\operatorname{sgn}\left(\Im\left[x_{k}\right]\right) \Im\left[\mathbf{h}_{k}^{T} \mathbf{d}\right]$. The BER of the quadrature-phase component for the $k$ th user, denoted as $P_{e_{Q}, k}(\mathbf{d})$, can be derived by replacing $c_{R}^{(k)}$ with $c_{I}^{(k)}$ in (25). Then the average BER for the quadrature-phase component of $\mathbf{y}$ is given by

$$
P_{e_{Q}, \mathbf{x}}(\mathbf{d})=\frac{1}{K} \sum_{k=1}^{K} P_{e_{Q}, k}(\mathbf{d})
$$

The resultant average BER of $\mathbf{y}$ is given by

$$
P_{e, \mathbf{x}}(\mathbf{d})=\left(P_{e_{I}, \mathbf{x}}(\mathbf{d})+P_{e_{Q}, \mathbf{x}}(\mathbf{d})\right) / 2
$$

Hence, the optimal effective symbol vector $\mathbf{d}_{\mathrm{opt}}$ is found by solving the following optimisation problem

$$
\mathbf{d}_{\mathrm{opt}}=\arg \min _{\mathbf{d}} P_{e, \mathbf{x}}(\mathbf{d})
$$

The problem (29) turns out to be a challenging nonconvex optimisation with many local minima. As an illustration, Figure 7 depicts the BER surface $P_{e, \mathbf{x}}(\mathbf{d})$ for the simplest case of $N=1$ and $K=1$, with $\mathrm{SNR}=16 \mathrm{~dB}$. The PSO algorithm of Section 2 offers an efficient means to solve this optimisation problem, where the cost function is $P_{e, \mathbf{x}}(\mathbf{d})$ with the parameter vector $\mathbf{d} \in \mathrm{U}^{N}$. For the system given in Subsection 4.1, our empirical results suggested that $U_{\max }=1.2$ and $V_{\max }=0.2$ are appropriate. The inertia weight is chosen as $\xi=\operatorname{rand}()$, which is seen to perform better in this application than the two alternative choices of $\xi$. In Step $a$ ), one of the initial particles is set to the improved MMSE-VP solution of. ${ }^{50}$ The two PSO algorithmic parameters that determine the performance and complexity of this PSO-aided MBER generalised VP design are then the swarm size $S$ and the maximum iteration number $I_{\max }$. Computation complexity analysis for this proposed MBER generalised VP scheme as well as for the existing MMSE VP design of ${ }^{45}$ can be found in. ${ }^{49}$

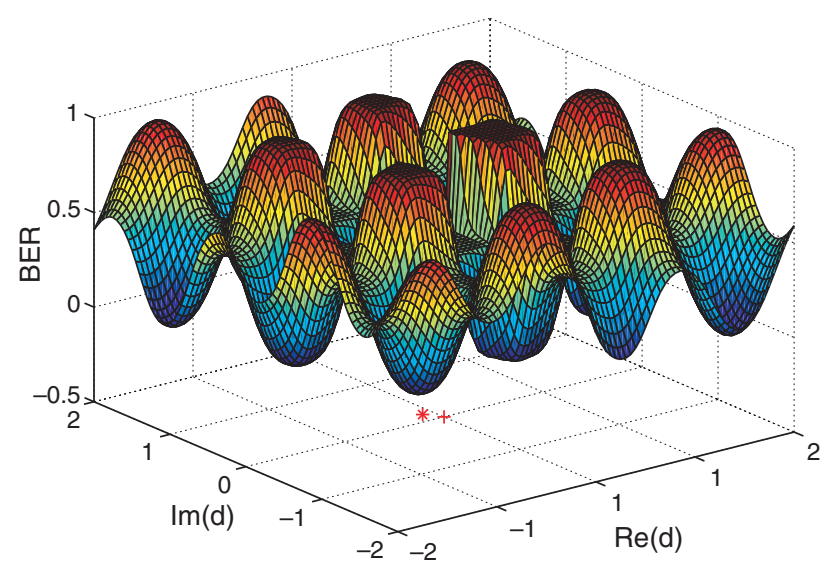

Fig. 7. BER surface as a function of the effective symbol vector $\mathbf{d}$ for the 4-QAM system with $N=1$ and $K=1$, given $\mathrm{SNR}=16 \mathrm{~dB}$. The mark * is the MBER generalised VP solution while the mark + is the MMSE VP solution. 


\subsection{Simulation Results}

We consider the challenging MIMO system, where the BS employed $N=2$ transmitter antennas to communicate with $K=4$ single-antenna MSs. This system was rank deficient as the number of the BS's transmitting antennas was smaller than the number of MSs supported. Again, all the simulation results were obtained by averaging over 100 channel realisations. The received signals after the modulo operation were directly used for making decisions. Appropriate swarm size was found empirically to be $S=20$, and the maximum number of iterations was ranging from $I_{\max }=20$ to 45 depending on the SNR value. Figure 8 shows the BER performance of the linear MBER-MUT design presented in Section 3, the powerful nonlinear MMSE-VP design presented in, ${ }^{45}$ and the proposed PSO-aided MBER generalised VP design. The linear MBER MUT encountered a high error floor as it was unable to differentiate the users' information in this demanding scenario. The nonlinear MMSE VP scheme showed a much better performance but still suffered from an error floor as can be seen in Figure 8. By contrast, the generalised MBER VP outperformed the MMSE VP and it did not exhibit a visible error floor which showed its ability to operate successfully in the rank-deficient scenario.

Convergence performance of the PSO-aided MBER generalised VP scheme with different swarm sizes and given $\mathrm{SNR}=25 \mathrm{~dB}$ are depicted in Figure 9. The results of Figure 9 show that $S=10$ was insufficient for the PSO algorithm to attain the global optimal solution, while the PSO algorithm with $S=20,30$ and 40 all converged to the optimal solution with $I_{\max }=40,32$ and 25 , respectively. The computational complexity $C_{\mathrm{PSO}}$ for the PSO algorithm with $S=20,30$ and 40 are compared in Table III, which

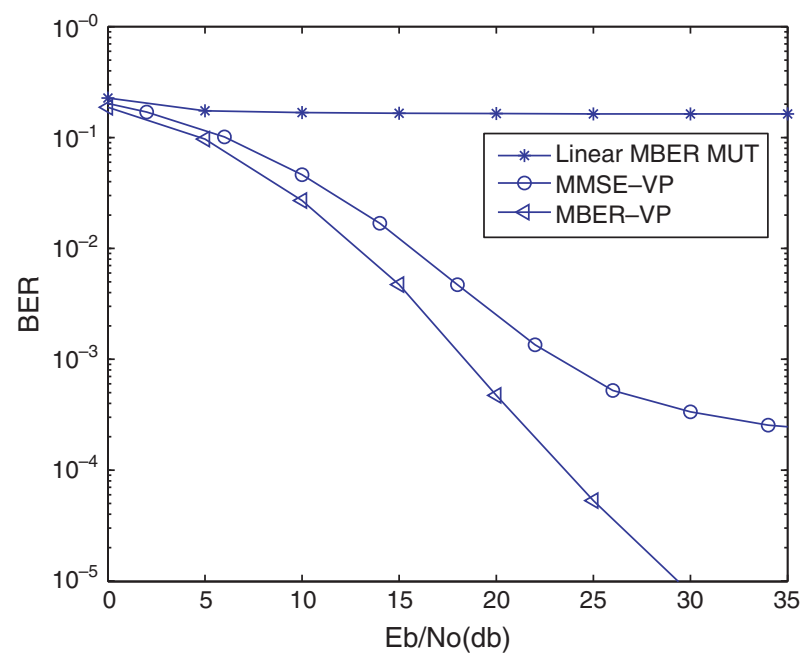

Fig. 8. Performance comparison of the linear MBER-MUT, the nonlinear MMSE-VP and the proposed PSO-aided MBER generalised VP for the $2 \times 4$ MIMO system.

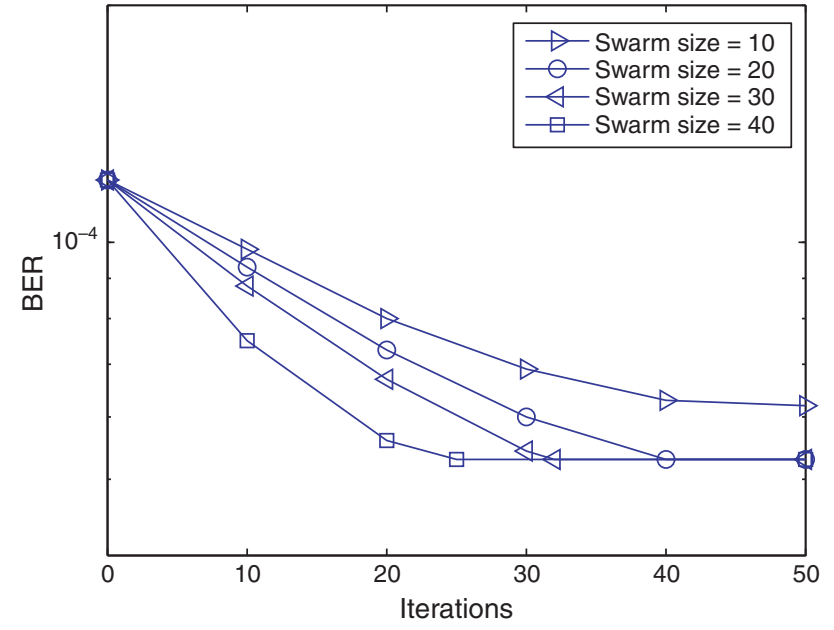

Fig. 9. Convergence of the PSO-aided MBER generalised VP scheme with different swarm sizes for the $2 \times 4$ MIMO system given $\mathrm{SNR}=$ $25 \mathrm{~dB}$.

Table III. Complexity (Flops) of the PSO aided MBER generalised VP design with different swarm sizes for the $2 \times 4$ MIMO system, given $\mathrm{SNR}=25 \mathrm{~dB}$.

\begin{tabular}{lcc}
\hline Swarm size $S$ & Iterations $I_{\max }$ & Complexity (Flops) \\
\hline 20 & 40 & $4,064,937$ \\
30 & 32 & $4,149,627$ \\
40 & 25 & $4,174,077$ \\
\hline
\end{tabular}

demonstrated that the choice of the swarm size $S=20$ for the PSO algorithm was optimal in terms of complexity in this case and explained why we used $S=20$ in the simulation. The computational complexity (Flops) as well as the recorded run times (s) of the two nonlinear MUT designs, namely the powerful MMSE-VP solution ${ }^{45}$ and the proposed PSO-aided MBER generalised VP solution, are compared in Table IV, given the two SNR values. It can be seen from Table IV that the complexity of the PSO aided MBER generalised VP design was no more than twice of the conventional MMSE-VP design. This was a small price worthy of paying, considering the significant performance enhancement of the former over the latter as shown in Figure 8.

Table IV. Complexity (Flops) and recorded run time (s) required by the MMSE-VP design and the PSO-aided MBER generalised VP design for the $2 \times 4$ MIMO system given two SNR values.

\begin{tabular}{lll}
\hline$(\mathrm{SNR}=25 \mathrm{~dB})$ & MMSE-VP & MBER-VP \\
\hline Complexity (Flops) & $2,508,638$ & $4,064,937$ \\
Run time (s) & 4787.3 & 8878.9 \\
(SNR $=30 \mathrm{~dB})$ & MMSE-VP & MBER-VP \\
Complexity (Flops) & $2,609,600$ & $4,471,060$ \\
Run time (s) & 4981.9 & 9565.8 \\
\hline
\end{tabular}

J. Comput. Theor. Nanosci. 9, 1-10, 2012 


\section{CONCLUSIONS}

PSO has been invoked for designing optimal MUT schemes for MIMO communication systems. Our investigation has demonstrated that PSO aided designs are capable of attaining global or near global optimal solutions at affordable computational costs. More specifically, the PSO aided linear MBER MUT scheme has been shown to impose significantly lower computational complexity than the existing state-of-the-art SQP-based linear MBER MUT design, while a novel PSO aided nonlinear MBER generalised VP design has been demonstrated to outperform the powerful nonlinear MMSE VP solution at the cost of slightly increased complexity.

\section{References}

1. S. Chen, Y. Wu, and S. McLaughlin, IEEE Trans. Evolutionary Computation 1, 259 (1997).

2. S. Chen and Y. Wu, IEEE Trans. Signal Processing 46, 1469 (1998).

3. K. Yen, Genetic Algorithm Assisted CDMA Multiuser Detection, $\mathrm{PhD}$ Thesis, School of Electronics and Computer Science, University of Southampton, Southampton, UK (2001).

4. M. Y. Alias, S. Chen, and L. Hanzo, IEEE Trans. Vehicular Technology 54, 1713 (2005).

5. W. Hua, Interference Suprression in Single- and Multi-Carrier CDMA Systems, PhD Thesis, School of Electronics and Computer Science, University of Southampton, Southampton, UK (2005).

6. M. Jiang, Hybrid Multi-user OFDM Uplink Systems Using Multiple Antennas, PhD Thesis, School of Electronics and Computer Science, University of Southampton, Southampton, UK (2005).

7. C. Xu, L.-L. Yang, and L. Hanzo, Ant-colony-based multiuser detection for MC DS-CDMA systems, Proceeding VTC'07-Fall, Baltimore, USA, September-October (2007), pp. 960-964.

8. C. Xu, L.-L. Yang, R. G. Maunder, and L. Hanzo, Near-optimum soft-output ant-colony-optimization based multiuser detection for the DS-CDMA, Proceeding ICC'08, Beijing, China, May (2008), pp. 795-799.

9. C. $\mathrm{Xu}, \mathrm{B}$. Hu, L.-L. Yang, and L. Hanzo, IEEE Trans. Vehicular Technology 57, 658 (2008).

10. J. Kennedy and R. Eberhart, Particle swarm optimization, Proceeding 1995 IEEE International Conference Neural Networks, Perth, Australia, November-December (1995), Vol. 4, pp. 1942-1948.

11. J. Kennedy and R. Eberhart, Swarm Intelligence, Morgan Kaufmann (2001).

12. A. Ratnaweera, S. K. Halgamuge, and H. C. Watson, IEEE Trans. Evolutionary Computation 8, 240 (2004).

13. S. M. Guru, S. K Halgamuge, and S. Fernando, Particle swarm optimisers for cluster formation in wireless sensor networks, Proceeding 2005 International Conference Intelligent Sensors, Sensor Networks and Information Processing, Melbourne, Australia, December (2005), pp. 319-324.

14. W. Fang, J. Sun, and W.-B. Xu, Design IIR digital filters using quantum-behaved particle swarm optimization, Proceeding 2nd International Conference Natural Computation, Xian, China, September (2006), Part II, pp. 637-640.

15. H.-M. Feng, Neurocomputing 70, 241 (2006).

16. J. Sun, W.-B. Xu, and J. Liu, Training RBF neural network via quantum-behaved particle swarm optimization, Proceeding ICONIP 2006, Hong Kong, China, October (2006), pp. 1156-1163.

17. S. Das and A. Konar, Engineering Applications of Artificial Intelligence 20, 1086 (2007).

18. F. A. Guerra and L. S. Coelho, Chaos, Solitons and Fractals 35, 967 (2008).
19. W.-F. Leong and G. G. Yen, IEEE Trans. Systems, Man and Cybernetics, Part B 38, 1270 (2008)

20. T.-Y. Sun, C.-C. Liu, T.-Y. Tsai, and S.-T. Hsieh, Adequate determination of a band of wavelet threshold for noise cancellation using particle swarm optimization, Proceeding CEC 2008, Hong Kong, China, June (2008), pp. 1168-1175.

21. M. S. Arumugam, G. R. Murthy, and C. K. Loo, Int. J. Bio-Inspired Computation 1, 198 (2009).

22. S. Chen, X. Hong, B. L. Luk, and C. J. Harris, Int. J. Bio-Inspired Computation 1, 246 (2009).

23. Q. M. Liu and W. Lv, Int. J. Bio-Inspired Computation 1, 270 (2009).

24. S. N. Sivanandam and P. Visalakshi, Int. J. Bio-Inspired Computation 1, 276 (2009).

25. J. Upendar, G. K. Singh, and C. P. Gupta, Int. J. Bio-Inspired Computation 2, 18 (2010).

26. J.-G. Lu, L. Zhang, H. Yang, and J. Du, Int. J. Bio-Inspired Computation 2, 27 (2010).

27. Z. Lu and S. Yan, Multiuser detector based on particle swarm algorithm, Proceeding 6th IEEE CAS Symposium Emerging Technologies: Frontiers of Mobile and Wireless Communication, Shanghai, China, May-June (2004), Vol. 2, pp. 783-786.

28. H. H. El-Mora, A. U. Sheikh, and A. Zerguine, Application of particle swarm optimization algorithm to multiuser detection in CDMA, Proceeding 16th IEEE International Symposium Personal, Indoor and Mobile Radio Communications, Berlin, Germany, September (2005), Vol. 4, pp. 2522-2526.

29. K. K. Soo, Y. M. Siu, W. S. Chan, L. Yang, and R.S. Chen, IEEE Trans. Vehicular Technology 56, 3006 (2007).

30. Z. Guo, Y. Xiao, and M. H. Lee, IEICE Trans. Communications E90-B, 421 (2007).

31. H. Liu and J. Li, IEEE Signal Processing Letters 15, 29 (2008)

32. W. Yao, S. Chen, S. Tan, and L. Hanzo, Particle swarm optimisation aided minimum bit error rate multiuser transmission, Proceeding ICC 2009, Dresden, Germany, June (2009), p 5.

33. L.-L. Yang, Design of linear multiuser transmitters from linear multiuser receivers, Proceeding ICC 2007, Glasgow, UK, June (2007), pp. 5258-5263.

34. D. Yang, L.-L. Yang, and L. Hanzo, Performance of SDMA systems using transmitter preprocessing based on noisy feedback of vectorquantized channel impulse responses, Proceeding VTC2007-Spring, Dublin, Ireland, April (2007), pp. 2119-2123.

35. B. R. Vojčić and W. M. Jang, IEEE Trans. Communications 46, 1346 (1998).

36. R. Irmer, R. Habendorf, W. Rave, and G. Fettweis, Nonlinear multiuser transmission using multiple antennas for TDD-CDMA, Proceeding 6th International Symposium Wireless Personal Multimedia Communications, Yokosuka, Japan, October (2003), pp. 251-255.

37. A. Hjørungnes and P. S. R. Diniz, IEEE Signal Processing Letters 12, 234 (2005).

38. R. Habendorf and G. Fettweis, Nonlinear optimization for the multiuser downlink, Proceeding 13th European Wirelss Conference, Paris, France, April (2007).

39. F. Richter, A. Fischer, R. Habendorf, and G. Fettweis, Transmitterbased minimization of error rates in the downlink of wireless systems, Proceeding GLOBECOM 2008, New Orleans, Louisiana, November-December (2008).

40. S. Tan, Minimum Error Rate Beamforming Transceivers, $\mathrm{PhD}$ thesis, School of Electronics and Computer Science, University of Southampton, UK (2008).

41. J. Nocedal and S. J. Wright, Numerical Optimization, New York: Springer (1999).

42. M. Costa, IEEE Trans. Information Theory 29, 439 (1983).

43. C. B. Peel, B. M. Hochwald, and A. L. Swindlehurst, IEEE Trans. Communications 53, 195 (2005).

44. B. M. Hochwald, C. B. Peel, and A. L. Swindlehurst, IEEE Trans. Communications 53, 537 (2005). 
45. D. A. Schmidt, M. Joham, and W. Utschick, Minimum mean square error vector precoding, Proceeding PIMRC 2005, Berlin, Germany, September (2005), Vol. 1, pp. 107-111.

46. E. Y. Kim and J. Chun, Optimum vector perturbation minimizing total MSE in multiuser MIMO downlink, Proceeding ICC 2006, Istanbul, Turkey, June (2006), Vol. 9, pp. 4242-4247.

47. W. S. Chua, C. Yuen, and F. Chin, A continuous vector-perturbation for multi-antenna multi-user communication, Proceeding VTC2007Spring, Dublin, Ireland, April (2007), pp. 1806-1810.
48. S. Chen, A. Livingstone, H.-Q. Du, and L. Hanzo, IEEE Trans. Wireless Communications 7, 1140 (2008).

49. W. Yao, S. Chen, and L. Hanzo, Generalised vector precoding design based on the MBER criterion for multiuser transmission, VTC 2010-Fall, Ottawa, Canada, September (2010), submitted

50. W. Yao, S. Chen, and L. Hanzo, Improved MMSE vector precoding based on the MBER criterion, Proceeding VTC2009-Spring, Barcelona, Spain, April (2009), p. 5.

Received: 30 June 2009. Accepted: 1 November 2009. 